\title{
The transient neutron star X-ray binary KS 1741-293 in outburst and quiescence
}

\author{
N. Degenaar ${ }^{1} \dagger$ and R. Wijnands ${ }^{2}$ \\ ${ }^{1}$ University of Michigan, Dept. of Astronomy, 500 Church St, Ann Arbor, MI 48109, USA \\ email: degenaar@umich.edu \\ ${ }^{2}$ Astronomical Institute "Anton Pannekoek", University of Amsterdam \\ Postbus 94249, 1090 GE Amsterdam, The Netherlands \\ email: r.a.d.wijnands@uva.nl
}

\begin{abstract}
KS 1741-293 is a transient neutron star low-mass X-ray binary that is located at an angular distance of $\simeq 20^{\prime}$ from the Galactic center. We map out the historic activity of the source since its discovery in 1989, characterize its most recent X-ray outbursts observed with Swift (2007, 2008, 2010, and 2011), and discuss its quiescent X-ray properties using archival Chandra data. KS 1741-293 is frequently active, exhibiting outbursts that typically reach a 2-10 keV luminosity of $L_{\mathrm{X}} \simeq 10^{36}(D / 6.2 \mathrm{kpc})^{2} \mathrm{erg} \mathrm{s}^{-1}$ and last for several weeks-months. However, Swift also captured a very short and weak accretion outburst that had a duration of $\lesssim 4$ days and did not reach above $L_{\mathrm{X}} \simeq 5 \times 10^{34}(D / 6.2 \mathrm{kpc})^{2} \mathrm{erg} \mathrm{s}^{-1}$. The source is detected in quiescence with Chandra at a $2-10 \mathrm{keV}$ luminosity of $L_{\mathrm{X}} \simeq 2.5 \times 10^{32}(D / 6.2 \mathrm{kpc})^{2} \mathrm{erg} \mathrm{s}^{-1}$.
\end{abstract}

Keywords. Accretion, accretion disks, stars: neutron, X-rays: binaries, X-rays: individual (KS 1741-293)

\section{Introduction}

KS 1741-293 is a transient neutron star low-mass X-ray binary (LMXB) that was discovered in 1989 August by the TTM onboard the KVANT module of the Mir space station (in 't Zand et al. 1991). The detection of type-I X-ray bursts revealed its binary nature and testified to the presence of a neutron star. The source is located at an angular distance of $\simeq 20^{\prime}$ from the Galactic center, at an estimated distance of $D \simeq 6.2 \mathrm{kpc}$ (as inferred from type-I X-ray burst analysis; Chelovekov \& Grebenev 2011). Throughout this work we assume a distance of $D \simeq 6.2 \mathrm{kpc}$ when quoting $\mathrm{X}$-ray luminosities.

\section{The outburst history of KS 1741-293}

We compiled a list of all (non-) detections of KS 1741-293 reported in literature, and combined these with the analysis of archival data (Sections 3 and 4) to map out its historic activity. Fluxes and upper limits were converted to the $2-10 \mathrm{keV}$ energy band using PIMMS, assuming a power law spectrum with $N_{\mathrm{H}}=21.5 \times 10^{22} \mathrm{~cm}^{-2}$ and $\Gamma=2.1$. The long-term X-ray light curve as observed between 1989-2012 is shown in Figure 1.

After its discovery in 1989, the field around KS 1741-293 was observed with ROSAT in 1992, but the source was not detected (Figure 1; Sidoli et al. 2001). Renewed activity was seen in 1998 March and September by BeppoSAX and $A S C A$, which possibly covered the same outburst (in 't Zand et al. 1998; Sidoli et al. 1999; Sakano et al. 2002). INTEGRAL detected it in outburst in 2003, 2004 and 2005 (de Cesare et al. 2007; Kuulkers et al. 2007; Chelovekov \& Grebenev 2011). Non-detections in between these epochs suggest

$\dagger$ Hubble fellow 


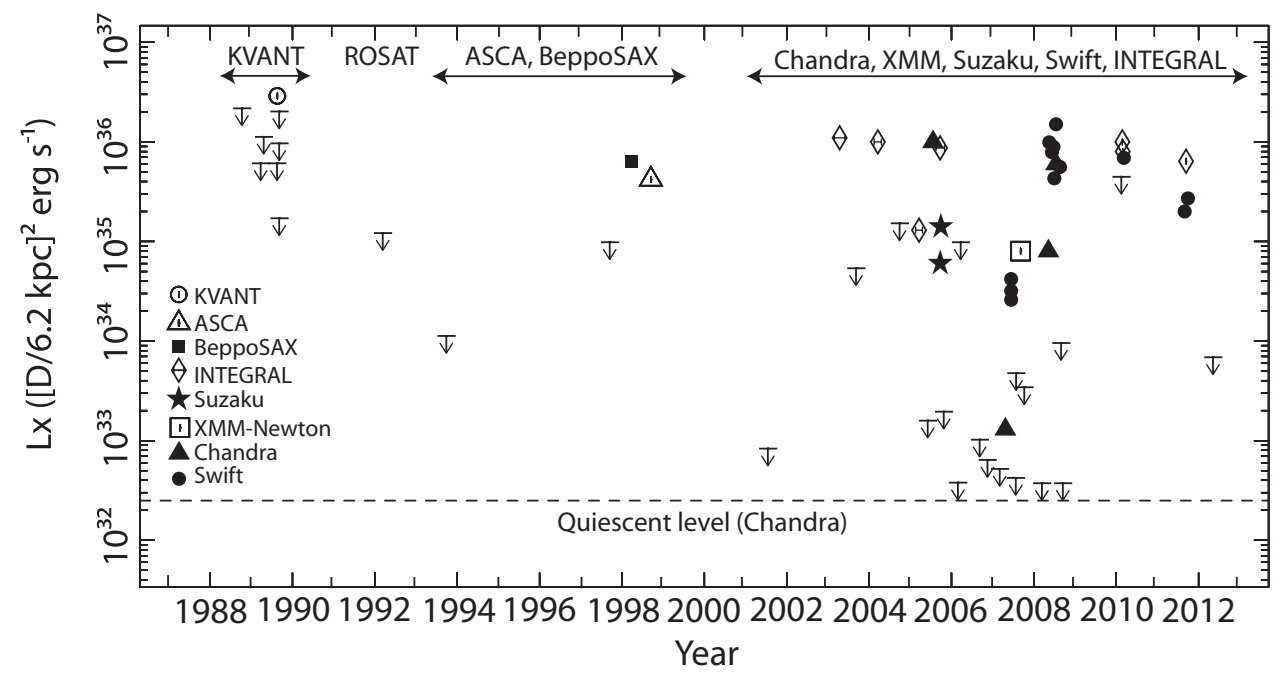

Figure 1. Long-term outburst history of KS 1741-293 (2-10 keV; see text for references).

that this were likely three separate outbursts (see Figure 1). The 2005 outburst was also observed by Chandra and Suzaku (Degenaar et al. 2008; Yuasa et al. 2008).

Between 2006 and 2008, the source region was covered by monitoring campaigns of the Galactic center with Chandra and XMM-Newton (Wijnands et al. 2006; Degenaar et al. 2012), and Swift (Degenaar \& Wijnands 2009, 2010). The source was found (weakly) active on three different epochs in 2007 (Section 3; Degenaar et al. 2012), and a new outburst was observed in 2008 (Degenaar \& Wijnands 2008; Degenaar et al. 2012). INTEGRAL and Swift found the source active again in 2010 (Chenevez et al. 2010) and in 2011 (Barthelmy et al. 2011; Linares et al. 2011; Chenevez et al. 2011).

Only a few type-I X-ray bursts have been reported for KS 1741-293. KVANT detected two (in 't Zand et al. 1991), six have been seen with INTEGRAL (de Cesare et al. 2007; Chelovekov \& Grebenev 2011), and recently one was picked up by Swift (Barthelmy et al. 2011; Linares et al. 2011). RXTE may have also detected one (Galloway et al. 2008).

\section{Outbursts observed with Swift between 2007 and 2012}

We obtained all Swift/XRT data covering KS 1741-293 from the public data archive. This encompasses 69 observations performed between 2007 May 5 and 2012 May 17. We extracted data products using the online XRT tools (Evans et al. 2009). Figure 2 displays the Swift/XRT light curve. It shows three major outbursts in 2008, 2010, and 2011, as well as a short and weak episode of activity in 2007. We extracted spectra for these four different outbursts and fitted these simultaneously in XSPEC to a simple absorbed power law model. The results of the spectral analysis are summarized in Table 1.

The region around KS 1741-293 was observed every few days by Swift between 2007 May 23 and August 9. The source is not detected during this epoch, except during three observations performed on June 13 and 14, when it displayed a 2-10 keV luminosity of $L_{\mathrm{X}} \simeq 3 \times 10^{34} \mathrm{erg} \mathrm{s}^{-1}$. Non-detections in the preceding and subsequent observations (June 11 and 15) suggest that this episode of low-level activity had a duration of $\lesssim 4$ days.

KS 1741-293 was detected at similarly low intensities at another two epochs in 2007. $X M M-N e w t o n$ found the source active at $L_{\mathrm{X}} \simeq 8 \times 10^{34} \mathrm{erg} \mathrm{s}^{-1}$ on 2007 September 6 . Non-detections with Swift on August 9 and September 27 constrain the duration of this 


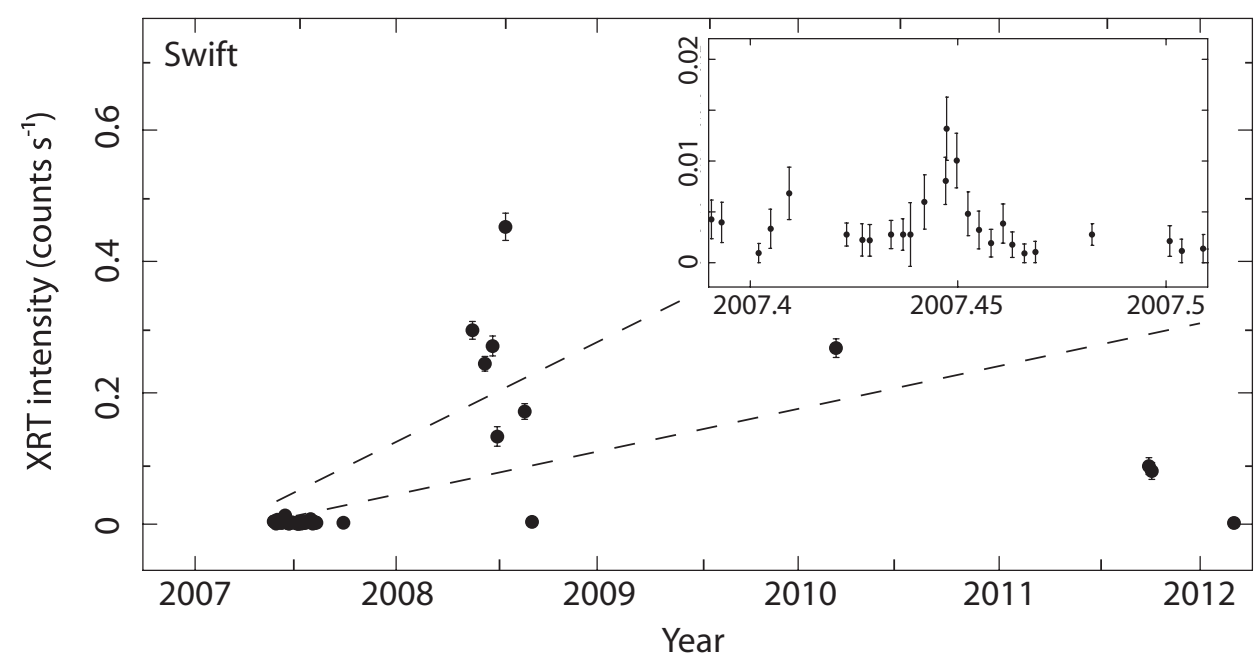

Figure 2. Long-term Swift/XRT light curve (binned per observation) showing three main outbursts of KS 1741-293 (2008, 2010, and 2011) and one episode of low-level activity (2007).

outburst to be $\lesssim 49$ days. In an archival Chandra observation performed on 2007 April 25 , KS 1741-293 is weakly detected at $L_{\mathrm{X}} \simeq 1.3 \times 10^{33} \mathrm{erg} \mathrm{s}^{-1}$ (Table 1). Non-detections with Chandra on 2007 February $19\left(L_{\mathrm{X}} \lesssim 5 \times 10^{32} \mathrm{erg} \mathrm{s}^{-1}\right)$ and Swift between 2007 May 23 and June $10\left(L_{\mathrm{X}} \lesssim 1 \times 10^{33} \mathrm{erg} \mathrm{s}^{-1}\right)$ suggest that the activity lasted for $\lesssim 3$ months. KS 1741-293 was thus found active a factor $\simeq 10-100$ above its quiescent level several times in 2007, but was not detected above $L_{\mathrm{X}} \simeq 10^{35} \mathrm{erg} \mathrm{s}^{-1}$.

We obtained a series of Swift/XRT ToO observations of KS 1741-293 between 2008 May 18 and September 4 to monitor the new outburst that was first detected by Chandra on May 10 (Degenaar et al. 2008; Degenaar \& Wijnands 2008). The source remained active for $\simeq 3$ months at an average 2-10 intensity of $L_{\mathrm{X}} \simeq 8 \times 10^{35} \mathrm{erg} \mathrm{s}^{-1}$ (Table 1) till August 21. It was no longer detected on September 4 with an upper limit of $L_{\mathrm{X}} \lesssim$ $9 \times 10^{33} \mathrm{erg} \mathrm{s}^{-1}$. In 2010 and 2011, KS 1741-293 was again observed in outburst with Swift at an intensity of $L_{\mathrm{X}} \simeq(3-7) \times 10^{35} \mathrm{erg} \mathrm{s}^{-1}$ (Table 1, Figure 2).

\section{KS 1741-293 in quiescence}

To investigate the quiescent properties, we used archival Chandra observations performed in 2001, 2006 and 2007 (Obs IDs 2267, 7038, and 8459; from the campaign of Muno et al. 2009). The source is not detected in the individual images, but clearly visible when the observations are combined $(\simeq 51 \mathrm{ks})$. We created a combined spectrum using the CIAO tools, and fitted this simultaneously with the Swift outburst data (Table 1).

KS 1741-293 is detected in quiescence at a $2-10 \mathrm{keV}$ luminosity of $L_{\mathrm{X}} \simeq 2.5 \times$ $10^{32} \mathrm{erg} \mathrm{s}^{-1}$, which is typical for quiescent neutron star LMXBs. The very large extinction in the direction of the source $\left(N_{\mathrm{H}} \simeq 2 \times 10^{23} \mathrm{~cm}^{-2}\right)$ completely obscures the thermal emission that is often detected for quiescent neutron star LMXBs (typically $k T_{\mathrm{bb}} \simeq 0.1-0.3 \mathrm{keV}$ ). Therefore, we only detect the hard (non-thermal) power law tail.

\section{Discussion}

KS 1741-293 is a frequently active: between 1989 and 2012, the source exhibited at least 8 accretion outbursts that reached $L_{\mathrm{X}} \simeq 10^{36} \mathrm{erg} \mathrm{s}^{-1}(2-10 \mathrm{keV})$ and had a duration 
Table 1. Spectral properties of KS 1741-293 in outburst and quiescence.

\begin{tabular}{cccccc}
\hline Instr. & Date & State & $\Gamma$ & $F_{\mathrm{X}}\left(\mathrm{erg} \mathrm{cm}^{-2} \mathrm{~s}^{-1}\right)$ & $L_{\mathrm{X}}\left(\mathrm{erg} \mathrm{s}^{-1}\right)$ \\
\hline Chandra & 2001/2006/2007 & quiescence & $1.3_{-1.3}^{+5.4}$ & $(5.5 \pm 5.0) \times 10^{-14}$ & $(2.5 \pm 2.3) \times 10^{32}$ \\
Chandra & 2007 Apr 25 & low activity & $0.2_{-0.2}^{+1.6}$ & $(2.8 \pm 1.3) \times 10^{-13}$ & $(1.3 \pm 0.6) \times 10^{33}$ \\
Swift & 2007 Jun 13-14 & low activity & $0.8_{-0.8}^{+2.4}$ & $(6.2 \pm 1.4) \times 10^{-12}$ & $(2.9 \pm 0.6) \times 10^{34}$ \\
Swift & 2008 May 18-Aug 21 & outburst & $2.1 \pm 0.4$ & $(1.7 \pm 0.4) \times 10^{-10}$ & $(7.8 \pm 1.9) \times 10^{35}$ \\
Swift & 2010 Mar 10 & outburst & $2.2 \pm 0.6$ & $(1.5 \pm 0.5) \times 10^{-10}$ & $(6.9 \pm 2.3) \times 10^{35}$ \\
Swift & 2011 Sep 1-30 & outburst & $2.7 \pm 1.6$ & $(6.0 \pm 2.4) \times 10^{-11}$ & $(2.8 \pm 1.1) \times 10^{35}$ \\
\hline
\end{tabular}

Note. Quoted errors refer to $90 \%$ confidence levels. A simultaneous fit to the spectral data resulted in $N_{\mathrm{H}}=(21.5 \pm 2.8) \times 10^{22} \mathrm{~cm}^{-2}$ and $\chi_{\nu}^{2}=1.1$ for 142 dof. $F_{\mathrm{X}}$ represents the unabsorbed $2-10 \mathrm{keV}$ flux and $L_{\mathrm{X}}$ the corresponding luminosity assuming $D=6.2 \mathrm{kpc}$.

of several weeks-months. This suggests a recurrence time of $\simeq 2$ yr and a duty cycle of $\simeq 12.5 \%$. In addition to these main outbursts, we found indications of low-level accretion activity a factor of $\simeq 10-100$ above the quiescent level of $L_{\mathrm{X}} \simeq 2.5 \times 10^{32} \mathrm{erg} \mathrm{s}^{-1}$. Such peculiar behavior has now been observed for a number of transient neutron star LMXBs, such as XMM J174457-2850.3, GRO J1744-28, GRS 1741-2853, and XTE J1701-462 (Degenaar \& Wijnands 2009, 2010; Degenaar et al. 2012; Fridriksson et al. 2011).

\section{Acknowledgements}

ND is supported by NASA through Hubble Postdoctoral Fellowship grant number HST-HF-51287.01-A from the Space Telescope Science Institute, and RW by a European Research Council starting grant. This work made use of Swift data supplied by the UK Swift Science Data Centre at the University of Leicester, and the Chandra data archive.

\section{References}

Barthelmy, S. D., Campana, S., D'Elia, V., et al. 2011, GRB Coordinates Network, 12319

Chelovekov, I. V. \& Grebenev, S. A. 2011, Astronomy Letters, 37, 597

Chenevez, J., Brandt, S., Kuulkers, E., et al. 2011, The Astronomer's Telegram, 3646

Chenevez, J., Kuulkers, E., Alfonso-Garzón, J., et al. 2010, The Astronomer's Telegram, 2465

de Cesare, G., Bazzano, A., Martínez Núñez, S., et al. 2007, MNRAS, 380, 615

Degenaar, N. \& Wijnands, R. 2008, The Astronomer's Telegram, 1541

Degenaar, N. \& Wijnands, R. 2009, A\&BA, 495, 547

Degenaar, N. \& Wijnands, R. 2010, A\&A, 524, A69

Degenaar, N., Wijnands, R., Cackett, E. M., et al. 2012, A\&A, 545, A49

Degenaar, N., Wijnands, R., \& Muno, M. 2008, The Astronomer's Telegram, 1531

Evans, P. A., Beardmore, A. P., Page, K. L., et al. 2009, MNRAS, 397, 1177

Fridriksson, J. K., Homan, J., Wijnands, R., et al. 2011, ApJ, 736, 162

Galloway, D. K., Muno, M. P., Hartman, et al. 2008, ApJS, 179, 360

in 't Zand, J., Heise, J., Smith, M., et al. 1998, IAU Circ., 6840, 2

in 't Zand, J. J. M., Heise, J., Brinkman, A. C., et al. 1991, Adv. Space Res., 11, 187

Kuulkers, E., Shaw, S. E., Paizis, A., et al. 2007, A\& $A, 466,595$

Linares, M., Chakrabarty, D., Cummings, J. R., et al. 2011, The Astronomer's Telegram, 3632

Muno, M. P., Bauer, F. E., Baganoff, F. K., et al. 2009, ApJS, 181, 110

Sakano, M., Koyama, K., Murakami, et al. 2002, ApJS, 138, 19

Sidoli, L., Belloni, T., \& Mereghetti, S. 2001, A\& A, 368, 835

Sidoli, L., Mereghetti, S., Israel, G. L., et al. 1999, ApJ, 525, 215

Wijnands, R., in 't Zand, J. J. M., Rupen, M., et al. 2006, A\&AA, 449, 1117

Yuasa, T., Tamura, K., Nakazawa, K., et al. 2008, PASJ, 60, 207 